\title{
Case Report \\ Case of "Slow" Stroke from Carotid Artery Occlusion Treated by Delayed but Cautious Endovascular Intervention
}

\author{
Kachi Illoh, ${ }^{1}$ Emilio Supsupin, ${ }^{2}$ Hashem M. Shaltoni, ${ }^{3}$ and Edwin D. Cacayorin ${ }^{2}$ \\ ${ }^{1}$ Division of Neurology Products, Center for Drug Evaluation and Research, U. S. Food and Drug Administration, Building 22, \\ Room 5402, 10903 New Hampshire Avenue, Silver Spring, MD 20993, USA \\ ${ }^{2}$ Department of Diagnostic and Interventional Imaging, The University of Texas Medical School at Houston, 6431 Fannin Street, \\ MSB 2.130B, Houston, TX 77030, USA \\ ${ }^{3}$ Department of Radiology, Baylor College of Medicine, One Baylor Plaza, Mail Stop BCM360, Houston, TX 77030, USA
}

Correspondence should be addressed to Kachi Illoh, kachikwu.illoh@fda.hhs.gov

Received 30 November 2010; Accepted 22 February 2011

Academic Editor: Osama O. Zaidat

Copyright (C) 2011 Kachi Illoh et al. This is an open access article distributed under the Creative Commons Attribution License, which permits unrestricted use, distribution, and reproduction in any medium, provided the original work is properly cited.

In a challenging case of carotid occlusion with slowly evolving stroke, we used brain imaging to facilitate endovascular revascularization resulting in the relief of the patient's symptoms. Patients with carotid occlusion and continued neurological worsening or fluctuations present enormous treatment challenges. These patients may present "slow" strokes with subacute infarcts that present significant challenges and risks during attempts at revascularization of the occluded artery. We present such a case in which we used multimodal imaging techniques, including MR-perfusion, to facilitate endovascular revascularization. Our approach of delayed but cautious intra-arterial thrombolytic therapy, guided by brain imaging, and followed by stent placement across the residual stenosis, enabled revascularization of the occluded artery without overt in-hospital complications.

\section{Introduction}

The management of ischemic stroke patients with acute carotid artery occlusion who experience neurological worsening is challenging and may require innovative approaches. The coexistence of carotid artery occlusion and evolving stroke symptoms poses major treatment challenges because of the presence of recent brain infarcts. Aggressive interventions with an aim of revascularization of the carotid artery are put on hold to avoid complications of reperfusion injury at the infarct sites. One such intervention, intra-arterial thrombolysis, is offered within six hours of onset in anterior circulation ischemic stroke [1]. Treatment beyond the 6hour window is associated with high risks of complications with limited chances of neurological improvement. In this report, we present an unusual case of a patient with acute carotid occlusion who developed neurological worsening and fluctuations ("slow" stroke) that required complex management decisions. Our approach was to perform delayed, but closely monitored, intra-arterial thrombolysis four days from initial stroke presentation. Our goal in presenting this case is to raise awareness of management challenges in the subacute phase of an ischemic stroke and highlight an interventional approach that may improve the patient's outcome. We show that delayed intra-arterial thrombolysis is achievable with improvement in the patient's condition and without untoward consequences.

\section{Clinical Summary}

The patient is a 66-year-old African American female who presented with repeated clinical events of brain ischemia and imaging confirmation of an infarct. She had a history of hypertension, type 2 diabetes mellitus, and hyperlipidemia. Initially, she presented to an outside hospital with a suddenonset of slurred speech that lasted 2 hours. She was diagnosed with transient ischemic attack (TIA) before discharge home on oral antiplatelet therapy. The following day, she developed another episode of slurred speech associated with left-sided weakness involving the face, arm, and leg. On presentation to the same outside facility, brain imaging revealed a right basal ganglia infarct with distal right internal carotid artery 
(ICA) stenosis. She was started on heparin infusion for possible dissection of the distal right ICA and transferred to our facility for a higher level of care. Her home medications included lisinopril, atorvastatin, long-acting insulin, rosiglitazone, and glyburide. Family history indicated her grandmother had a stroke of unclear cause. She did not smoke, drink alcohol, or use illicit drugs. Clearly, her hospital transfer indicated a deteriorating ischemic process of the brain that was referable to carotid artery pathology.

The patient's subsequent hospitalization was marked by episodes of neurological worsening. On transfer to our facility three days from her initial TIA symptoms, her blood pressure was $155 / 81 \mathrm{mmHg}$ and heart rate was 105 beats per minute (bpm). Neurological examination revealed a mild left facial droop with an NIH stroke scale (NIHSS) score of 1; other aspects of neurological and systemic examination were unremarkable. A head CT scan showed the right basal ganglia infarct, and CT angiography confirmed the ICA stenosis. She was started on antiplatelet therapy (clopidogrel) and discontinued heparin treatment. Within 10 hours of transfer, she developed the first neurological worsening in our facility. She had a partial right gaze preference, partial left visual field cut, more prominent left facial droop, and left arm drift (NIHSS 4). Her vital signs at this time indicated a drop in blood pressure to $115 / 55 \mathrm{mmHg}$, with a pulse rate of $79 \mathrm{bpm}$. She received additional normal saline infusion and was started on eptifibatide infusion. Seven hours from onset of worsening, she improved to the point of having no neurological deficits (NIHSS 0). Her blood pressure at that time was $145 / 75 \mathrm{mmHg}$ with pulse rate of $77 \mathrm{bpm}$. The next day (four days from initial TIA symptoms), we performed a cerebral angiogram to better define her vasculature and identified a right carotid terminus (carotid $\mathrm{T}$ ) occlusion. The right anterior cerebral artery (ACA) was perfused from the anterior communicating (A-Comm) artery while the right middle cerebral artery (MCA) territory was perfused by leptomeningeal collaterals and right posterior cerebral artery (PCA). No evidence of carotid artery dissection was found. No interventional revascularization procedure was attempted at this point given recent infarct and potential risks of reperfusion injury. Following the arteriography, she continued to have neurological fluctuations despite attempts to keep her blood pressure elevated. Each time she sat up for up to 15 minutes, she developed a left-face and arm weakness typically with a drop in systolic blood pressure to below $114 \mathrm{mmHg}$. Notably, she was remarkably frustrated (she felt miserable, saying she would rather die than continue to have these episodes) though her NIHSS score only worsened to a score of 4. For this patient, her level of psychological distress especially with inability to sit up was a major disability, a feature not captured by the severity scale of NIHSS.

An MRI showed extensive perfusion defects suggesting considerable risk of imminent brain damage, which prompted our subsequent endovascular intervention. We obtained additional investigative studies including electroencephalogram (EEG) and echocardiography (both transthoracic and transesophageal); the results of these were unremarkable. However, an MRI of the brain showed the right basal ganglia infarct with extensive perfusion defect in the right MCA territory (Figure 1) and no evidence of hemorrhagic transformation of infarct. These findings, in addition to the patient's clinical condition, prompted our endovascular intervention five days from her initial TIA event. With the patient under conscious sedation and being monitored for neurological status, we slowly infused urokinase through a microcatheter at site of occlusion (Figure 2(a)). After the intra-arterial infusion of 107,000 units of urokinase, the patency of the right ICA and MCA were reestablished; perfusion of the lateral lenticulostriate arteries became evident. However, an underlying severe stenosis remained at the distal right ICA bifurcation (Figure 2(b)). Again, due to concerns of reperfusion injury, it was elected to proceed with treatment of the residual stenosis at a later date. She remained free of neurological fluctuations following the procedure. Twelve days from her initial symptoms, a balloon-mounted, drug-eluting $2.5 \times$ $8 \mathrm{~mm}$ Cypher Stent (Cordis, Miami Lakes, Florida) was successfully deployed at the stenotic segment of the right supraclinoid ICA bifurcation. Complete recanalization was achieved with the procedure, enabling an improvement in ICA luminal diameter from $1 \mathrm{~mm}$ to $2.5 \mathrm{~mm}$ as shown in Figure 3. Following the procedure, she was maintained on an antiplatelet regimen of clopidogrel and aspirin to maintain stent patency and measures to keep her systolic blood pressure below $140 \mathrm{mmHg}$ to prevent hyperperfusion injury. The patient was discharged home the following day without neurological deficits or further episodes of worsening.

\section{Discussion}

This case is striking because it highlights the use of slowly delivered intra-arterial thrombolysis to revascularize an occluded carotid artery terminus in the subacute ischemic stroke period, followed by stenting of the residual stenosis. This management approach was necessitated by the continued neurological fluctuations despite conservative medical measures. Also, there was imaging suggestive of additional brain tissue at risk of further damage. This imaging information provided the basis for our interventional approach. The clinical presentation of our patient is unusual in that she had an acute large vessel occlusion at the carotid $\mathrm{T}$ and slowly developed very minimal neurological deficits accentuated with intermittent fluctuations. Acute carotid $\mathrm{T}$ occlusions are typically devastating with poor clinical outcomes. The manner of our patient's presentation, termed "slow" stroke, is invariably difficult to treat because of the risk of reperfusion injury. Therefore, our therapeutic approach was facilitated largely by serial brain imaging besides the clinical fluctuations exhibited by the patient. We used MR imaging to delineate the infarct and brain tissue at risk of dying (penumbra). The penumbra that persisted many days from onset of initial insult presented a window of opportunity for intervention. The presence of perfusion defect or penumbra as estimated on MRI appears to be a useful marker in identifying patients that could be treated beyond the hyperacute period [2-5]. However, these studies evaluated imaging markers of penumbra in patients within 


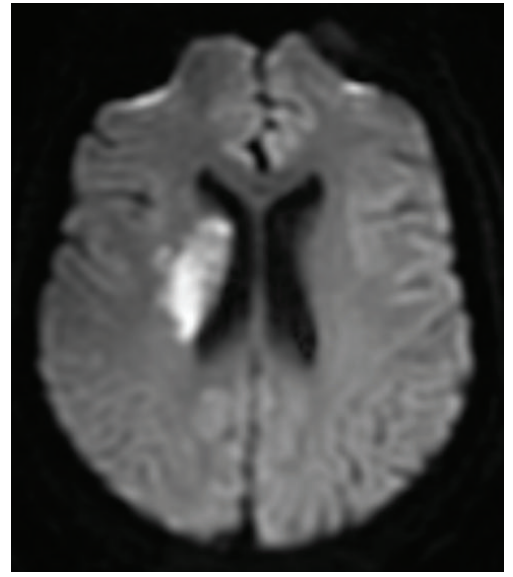

(a)

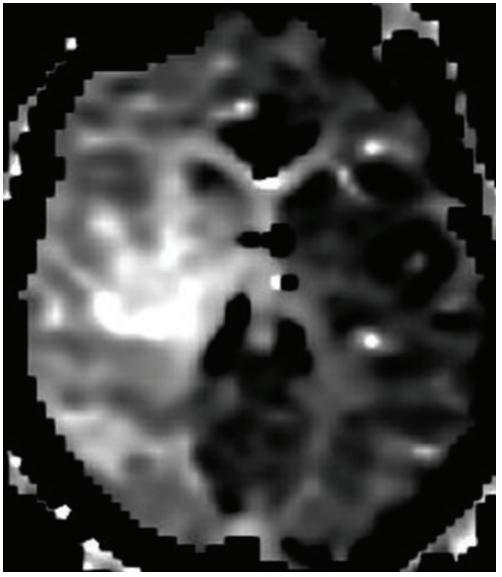

(b)

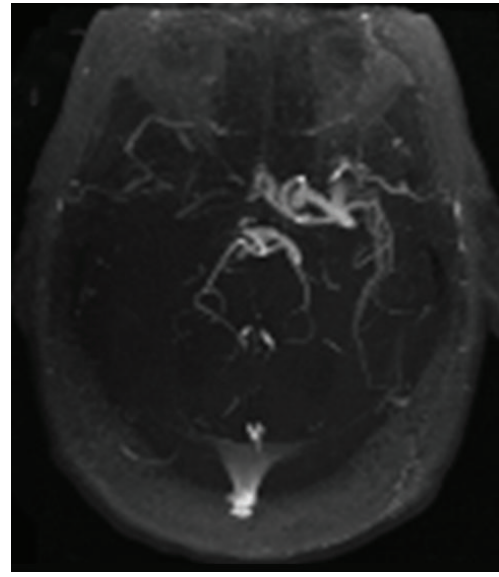

(c)

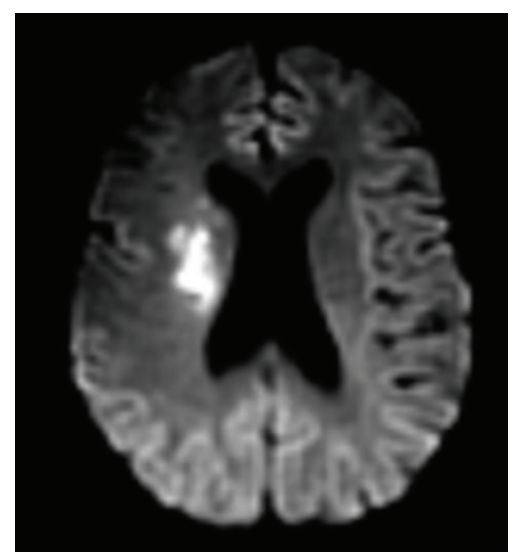

(d)

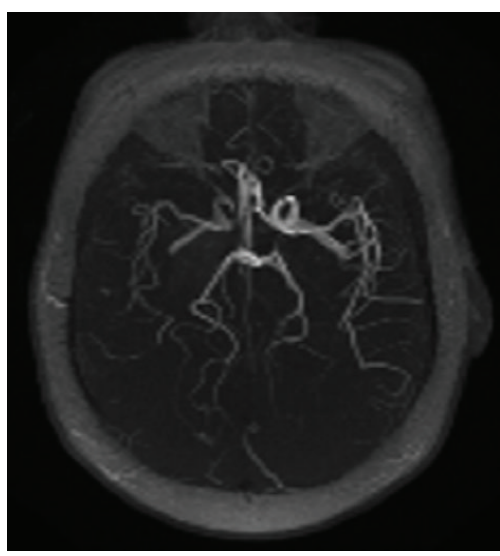

(e)

FIGURE 1: Magnetic resonance imaging of the brain, (a) prethrombolysis diffusion-weighted image (DWI) shows brain infarct in the right MCA territory, (b) prethrombolysis Perfusion-weighted image (PWI) shows right brain hemisphere with perfusion defect, (c) prethrombolysis Magnetic resonance Angiography (MRA) shows absence of blood flow in the right MCA, (d) postthrombolysis DWI shows no significant interval changes in brain parenchyma, and (e) postthrombolysis MRA with patent right MCA.

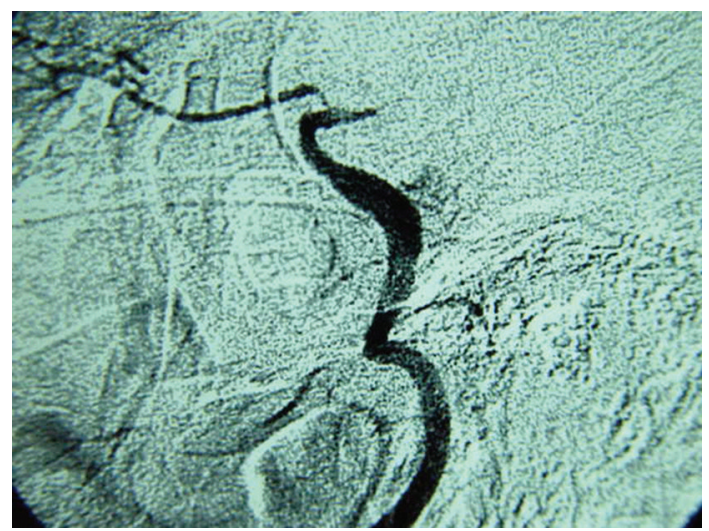

(a)

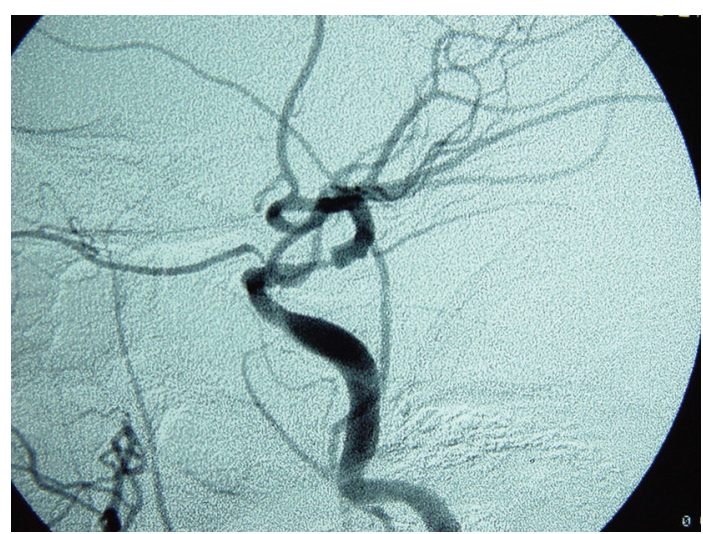

(b)

FIGURE 2: (a) Slow injection of dilute urokinase with microcatheter at site of occlusion; (b) recanalization of the right MCA with residual carotid stenosis. 


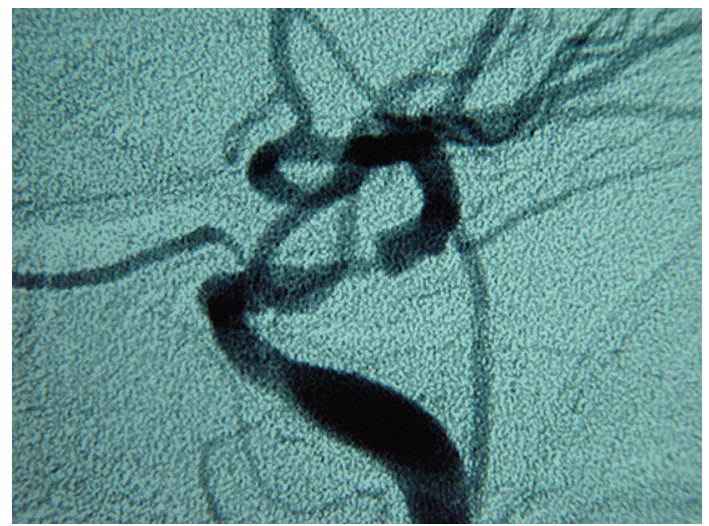

(a)

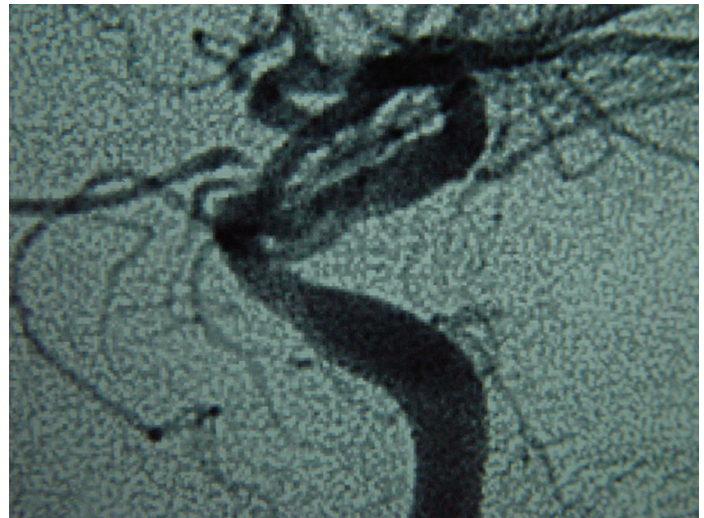

(b)

Figure 3: (a) Prestenting cerebral arteriography shows occluded terminal carotid artery; (b) Poststenting arteriography shows complete carotid recanalization.

6 hours of stroke onset and not days after as in our patient's case.

Our approach differs from those of others who have employed various means such as induction of hypertension. Medically induced hypertension improves brain perfusion and has been shown to ameliorate stroke symptoms beyond the thrombolysis window $[6,7]$. We were unable to stabilize our patient's condition with such conservative measures. Also, it is possible that medically induced hypertension using vasopressors on the long term could pose a risk of damage in other vascular beds given a patient's vascular risk factors [1]. Even so, keeping her hypertensive in hospital was difficult suggesting that continuation of such therapy as an outpatient would be impracticable. Had carotid dissection been confirmed on initial angiography, anticoagulation could have been an option of medical therapy. Nonetheless, we were unable to achieve neurological stability with medical therapy that consisted of antiplatelet therapy and attempts at keeping the patient hypertensive. Revascularization of acute carotid occlusion with thrombolysis and device-assisted approaches is promising in the acute stroke setting [8]. Complications of such revascularization (procedures and thrombolysis) and lack of palpable benefit limit the use of this therapeutic approach beyond the acute stroke period. Typically, it is unlikely that our patient would be a candidate for aggressive interventions to reopen the occluded carotid artery given her presentation with initial negligible deficits. No doubt, her development of neurological worsening despite conservative measures helped direct our therapeutic approach. With the risk of hemorrhage from thrombolysis, we considered mechanical thrombectomy as an alternative method of intervention. However, we wanted to avoid abrupt recanalization of the occluded artery that could precipitate reperfusion injury especially in the setting of a subacute infarct. Therefore, we decided on urokinase infusion with the goal of slow carotid recanalization. Although recent reports highlight the feasibility of delayed reperfusion modalities [9, $10]$, it remains to be determined whether our approach was the optimum in the clinical setting. A systematic evaluation of the approach, including long-term clinical outcomes, is needed before it can be recommended.

Other treatment options appeared to be of limited value in addressing the patient's misery from neurological fluctuations. Arterial bypass surgery would be unlikely to ameliorate the symptoms that early after the stroke. Moreover, its benefits in stroke patients remain unclear. Though not common, carotid endarterectomy (CEA) has been reportedly successful in some patients with acutely occluded ICA [11]. In our patient, the location of the carotid occlusion made such surgical revascularization untenable. In such tenuous vascular locations, revascularization using stent placement can be successfully achieved in patients with acute carotid occlusion and subacute infarcts [12]. In conclusion, we demonstrate that delayed and closely monitored intra-arterial therapy followed by stenting of residual atherosclerotic stenosis is achievable in a patient presenting with a subacute ischemic stroke and neurological fluctuations. In such a case, brain imaging showing evidence of substantial brain tissue at risk with absence of hemorrhagic transformation is useful in facilitating management decisions.

\section{Disclosure}

The views expressed in this paper are those of the authors and do not necessarily represent the official position of their employers, including the U.S. Food and Drug Administration.

\section{References}

[1] H. P. Adams, G. Del Zoppo, M. J. Alberts et al., "Guidelines for the early management of adults with ischemic stroke," Stroke, vol. 38, no. 5, pp. 1655-1711, 2007.

[2] J. J. Legos, S. C. Lenhard, R. E. Haimbach et al., "SB 234551 selective ET receptor antagonism: perfusion/Diffusion MRI used to define treatable stroke model, time to treatment and mechanism of protection," Experimental Neurology, vol. 212, no. 1, pp. 53-62, 2008. 
[3] S. M. Davis, G. A. Donnan, M. W. Parsons et al., "Effects of alteplase beyond $3 \mathrm{~h}$ after stroke in the Echoplanar Imaging Thrombolytic Evaluation Trial (EPITHET): a placebocontrolled randomised trial," The Lancet Neurology, vol. 7, no. 4, pp. 299-309, 2008.

[4] M. Köhrmann, E. Jüttler, H. B. Huttner, T. Nowe, and P. D. Schellinger, "Acute stroke imaging for thrombolytic therapyan update," Cerebrovascular Diseases, vol. 24, no. 2-3, pp. 161169, 2007.

[5] G. W. Albers, V. N. Thijs, L. Wechsler et al., "Magnetic resonance imaging profiles predict clinical response to early reperfusion: the diffusion and perfusion imaging evaluation for understanding stroke evolution (DEFUSE) study," Annals of Neurology, vol. 60, no. 5, pp. 508-517, 2006.

[6] T. Bogoslovsky, O. Häppölä, O. Salonen, and P. J. Lindsberg, "Induced hypertension for the treatment of acute MCA occlusion beyond the thrombolysis window: case report," BMC Neurology, vol. 6, article 46, 2006.

[7] A. E. Hillis, R. J. Wityk, N. J. Beauchamp, J. A. Ulatowski, M. A. Jacobs, and P. B. Barker, "Perfusion-weighted MRI as a marker of response to treatment in acute and subacute stroke," Neuroradiology, vol. 46, no. 1, pp. 31-39, 2004.

[8] T. Tomsick, J. Broderick, J. Carrozella et al., "Revascularization results in the interventional management of stroke II trial," American Journal of Neuroradiology, vol. 29, no. 3, pp. 582587, 2008.

[9] C. A. Molina and J. L. Saver, "Extending reperfusion therapy for acute ischemic stroke: emerging pharmacological, mechanical, and imaging strategies," Stroke, vol. 36, no. 10, pp. 2311-2320, 2005.

[10] A. Abou-Chebl, "Endovascular treatment of acute ischemic stroke may be safely performed with no time window limit in appropriately selected patients," Stroke, vol. 41, no. 9, pp. 1996-2000, 2010.

[11] B. T. Weis-Müller, R. Huber, A. Spivak-Dats, B. Turowski, M. Siebler, and W. Sandmann, "Symptomatic acute occlusion of the internal carotid artery: reappraisal of urgent vascular reconstruction based on current stroke imaging," Journal of Vascular Surgery, vol. 47, no. 4, pp. 752-759, 2008.

[12] K. Imai, T. Mori, H. Izumoto et al., "Successful stenting seven days after atherothrombotic occlusion of the intracranial internal carotid artery," Journal of Endovascular Therapy, vol. 13, no. 2, pp. 254-259, 2006. 


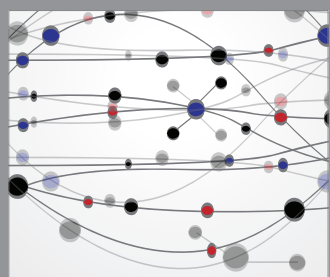

The Scientific World Journal
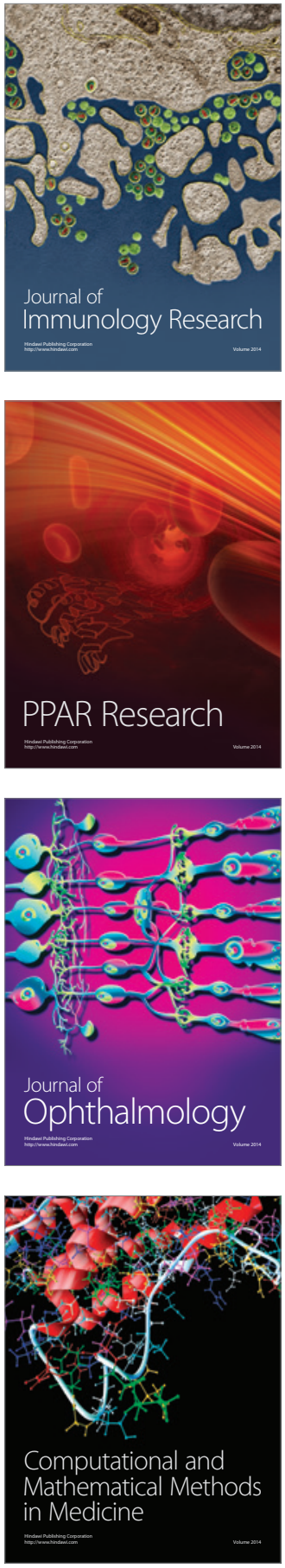

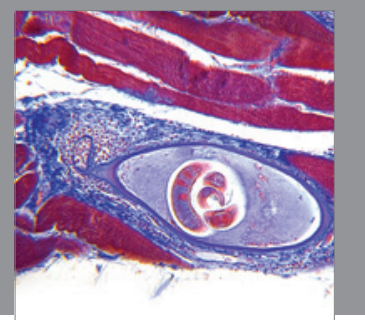

Gastroenterology

Research and Practice
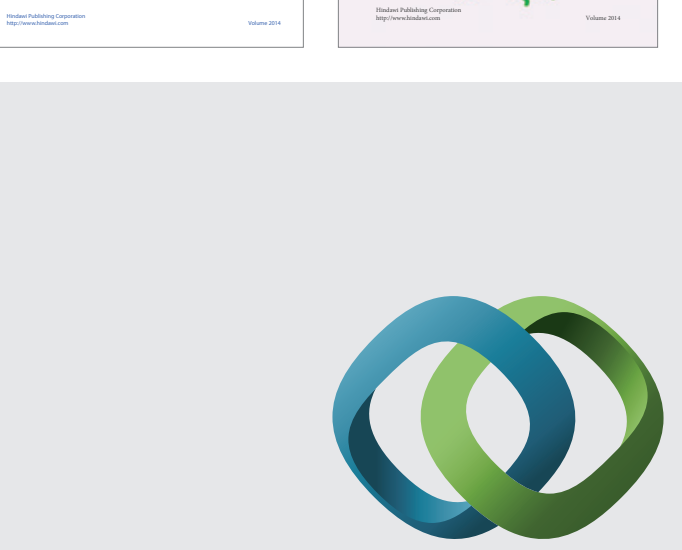

\section{Hindawi}

Submit your manuscripts at

http://www.hindawi.com
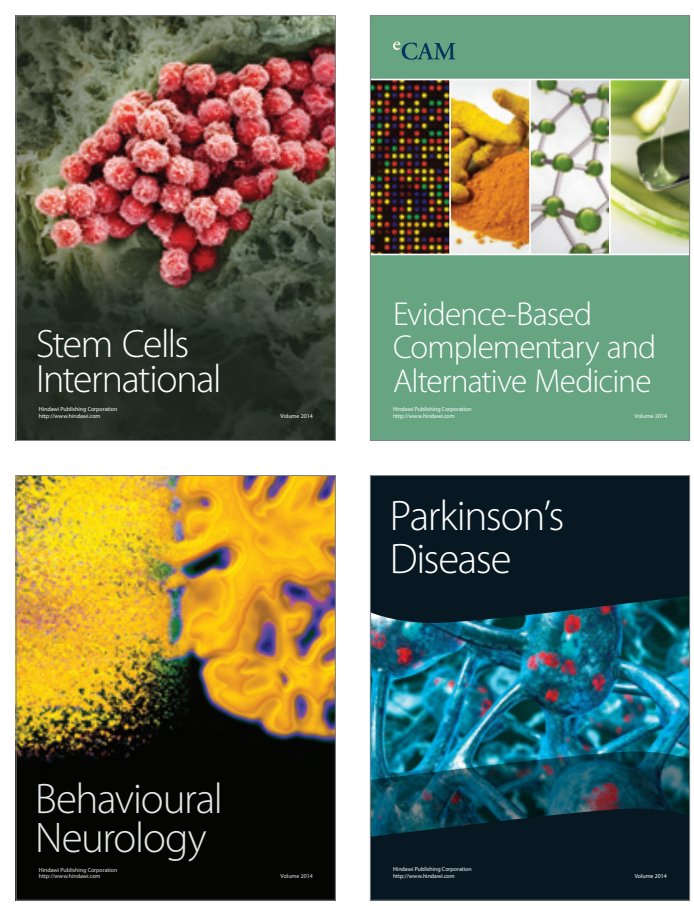

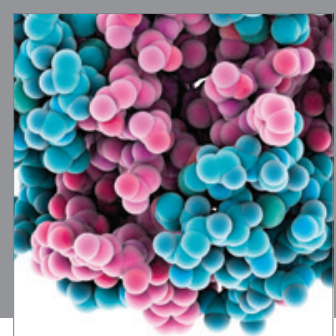

Journal of
Diabetes Research

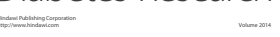

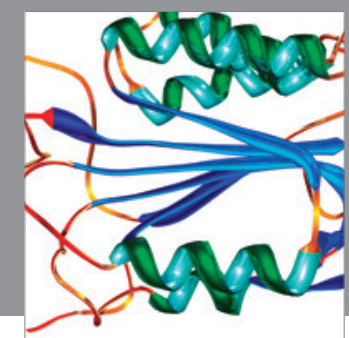

Disease Markers
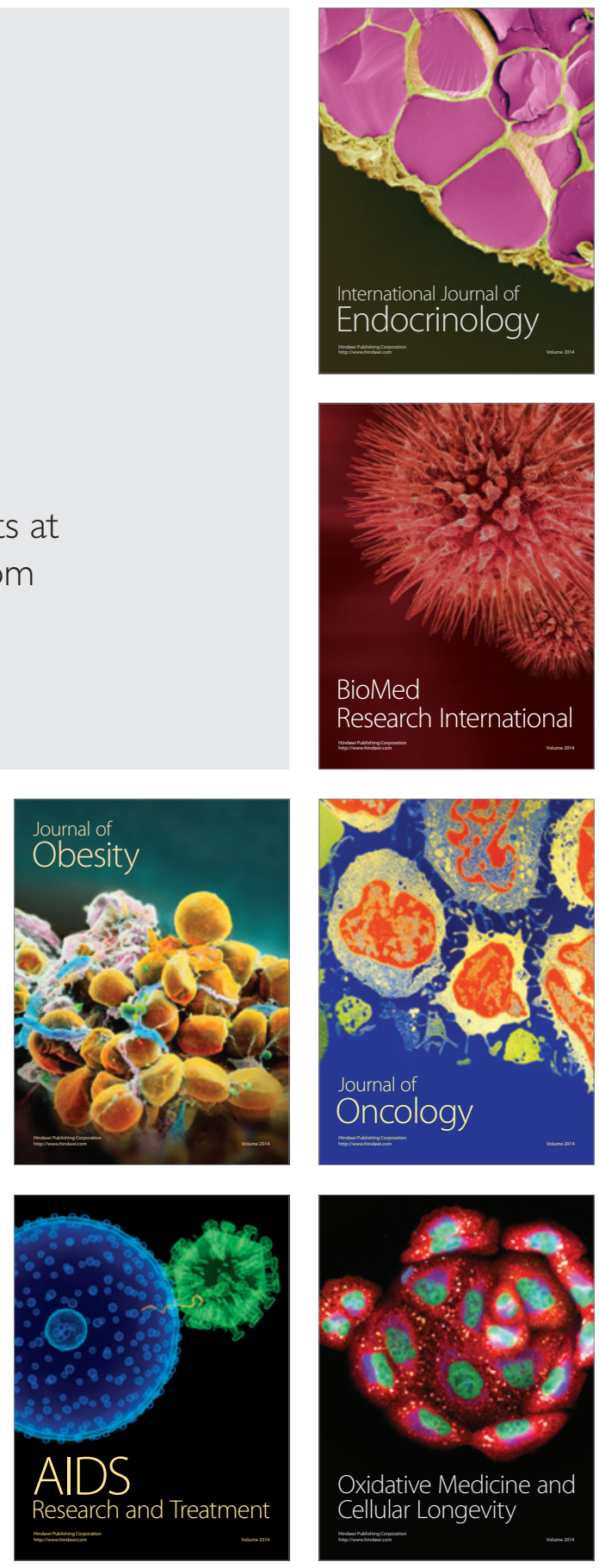